\title{
Direct Percutaneous Alcohol Sclerotherapy for Venous Malformations of Head and Neck Region without Fluoroscopic Guidance: Technical Consideration and Outcome
}

\author{
Hye Jin Baek, MD', Joon Pio Hong, MD², Jong Woo Choi, MD², Dae Chul Suh, MD'1
}

Purpose: Alcohol is not used directly to the vascular lesion without mixing with the contrast agent because alcohol itself cannot be seen on the fluoroscopy. Since we have used alcohol for the venous malformations in the head and neck area, we realized that alcohol can be safely and effectively used without using fluoroscopy. We present the method of direct sclerotherapy using absolute alcohol without using fluoroscopy.

Materials and Methods: After obtaining and carefully analyzing direct puncture venogram, we used this technique in 22 patients who underwent alcohol sclerotherapy. Because fluoroscopy was not used during alcohol injection, the angiotable can be placed outside of C-arm so that alcohol was comfortably injected without any obstacle around the patients. Venogram can also be obtained between the injections to detect whether there is any dangerous venous outflow drainage such as the superior ophthalmic vein to the cavernous sinus. To control the venous outflow, local compression to the draining vein was applied. The result and complication such as skin necrosis, infection, and nerve injury were evaluated during mean follow-up period of 13 months (range, 1-63 months).

Results: The frequency of sclerotherapy was one in 16 and 2-5 in 6 patients. The volume of alcohol used per treatment session ranged from 2 to $18 \mathrm{~mL}$ (mean, $8.5 \mathrm{~mL}$ ). There was the minimum change in 1 $(4.5 \%)$, moderately decreased lesion in $12(54.5 \%)$, and markedly decreased lesion in $9(41 \%)$ patients. The patients did not reveal any complications during 12.9 months follow-up period.

Conclusion: Direct puncture alcohol sclerotherapy without using fluoroscopy can be a safe and effective technique for treating venous malformation of the head and neck areas. In addition, the procedure can be performed in the comfortable position because biplane fluoroscopy would not be necessary.

Key Words : Vascular malformations; Sclerotherapy; Ethanol; Endovascular techniques

\footnotetext{
'Department of Radiology and Research Institute of Radiology, ${ }^{2}$ Department of Plastic Surgery, University of Ulsan, College of Medicine, Asan Medical Center, Seoul, Korea

Received February 7, 2011; accepted after revision May 4, 2011.

Correspondence to: Dae Chul Suh, MD, Department of Radiology, University of Ulsan, College of Medicine, Asan Medical Center, 86 Asanbyeongwon-gil, Songpa-gu, Seoul 138-736, Korea.

Tel. 82.2.3010.4366 Fax. 82.2.476.0090 E-mail: dcsuh@amc.seoul.kr

This is an Open Access article distributed under the terms of the Creative Commons Attribution Non-Commercial License (http://creativecommons.org/licenses/by-nc/3.0) which permits unrestricted non-commercial use, distribution, and reproduction in any medium, provided the original work is properly cited.
} 
Venous malformation (VM) is a common vascular malformation, although they are not always evident at birth, with $40 \%$ of cases found in the head and neck region $[1-3]$. And it has been known as one of the most difficult disease entities diagnostically and therapeutically in the daily practice $[4,5]$.

The treatment of VMs presents a challenge to interventional physicians. Percutaneous alcohol sclerotherapy has been reported to be effective in the primary treatment of venous malformations of the head and neck region [5-8]. Alcohol is believed to have the lowest rate of recurrence of all the sclerosants [7]. However, it is associated with potential clinically important complications that were usually classified as either local or systemic. Local complications include any transient adverse effects, such as transient nerve injuries, skin blistering, or skin necrosis. Systemic complications include death, and permanent adverse effects, such as cardiovascular or pulmonary events [4, $6,7,9]$. Therefore, a total amount of alcohol that can be given in a procedure should not exceed $1.0 \mathrm{~mL} / \mathrm{kg}$ body weight $[4,7,10,11]$. The dose limitation is based on the clinical experience of the interventional physicians with respect to the procedure itself and the postprocedural course [7]. However, systemic introduction of alcohol can be dangerous, regardless of dose used, for large VM with rapid inflow and outflow of the lesion. Thus, it is important to regulate flow factors of VM to achieve alcohol sclerotherapy safely and effectively.

In conventional percutaneous slcerotherapy, manual compression of the draining vein has been usually used for flow reduction to avoid inadvertent distal migration of sclerosant [12]. In contrast, we used pure absolute alcohol without mixing contrast agent and fluoroscopic guidance with manual compression to the VM itself. We found that this method can be helpful to increase sclerotic effect and to reduce the total amount of alcohol and complications. We present our experience and the outcome of percutaneous alcohol sclerotherapy without using fluoroscopy for VM of the head and neck region.

\section{MATERIALS AND METHODS}

\section{Patient Selection}

We obtained approval from the institutional review board of our hospital for a review and utilization of patient medical and imaging records. Written informed consent for the procedure was obtained from all patients and their families after a discussion about the advantages and risks for the procedure.

Patients were identified from the neurointerventional database of our institution. From May 2007 to June 2009, a total of 22 consecutive patients with VMs of the head and neck region were treated with absolute alcohol sclerotherapy (12 female patients and 10 male patients) in our department. The mean age was 30.3 years (age range, 3-67 years). The onset of the symptoms or detection of the lesion varied from childhood to adulthood. Nineteen of the lesions were located on the face, including the lip, cheek, submandibular, temporal, and periorbital areas. One of the lesions was on the tongue, and 2 were located in the neck. All patients had one or more symptoms and the most common symptom was painless swelling (95.5\%) with the next common symptom being discoloration (45.5\%). Two had recurrent bleeding episodes from the lesion.

Eleven patients had a history of operations, and the remaining 11 patients were treated by alcohol sclerotherapy as the primary treatment technique and then a patient out of 11 underwent surgical resection.

\section{Treatment Details}

Direct percutaneous alcohol sclerotherapy was performed under intermittent fluoroscopic guidance (Axiom Artis dBA system, Siemens AG, Medical Solutions, Forchheim, Germany), sterile conditions, and general anesthesia. 18-gauge needle (BD Angiocath Plus, Becton Dikinson Korea, Seoul, Korea) were directly inserted into the venous pouch of VM until blood return was observed. Venography with water-soluble iodinated contrast material confirmed needle position within the VM and assessed venous drainage. Appropriate venous pouch occlusion was performed with manual compression, and contrast material injection was repeated until no undesirable outflow into normal vessels occurred. This step was applied when the venous drainage was toward deep or dangerous/intracranial structures such as the cavernous sinus.

Alcohol was then injected under visual control for VMs without using fluoroscopic control. If required, fluoroscopic guidance was used between the alcohol injections to detect whether there is any dangerous venous outflow drainage such as the superior ophthalmic vein to the cavernous sinus. Up to $18 \mathrm{cc}$ of alcohol was injected at each session. Absolute (99\%) alcohol was used during the procedure without mixing with the contrast agent to render the mixture radiopaque. The total volume and the volume of the 
individual aliquots of alcohol were determined according to the size of the VM and the size of the vessels being injected, with a maximum total dose of 1.0 $\mathrm{mL} / \mathrm{kg}$. The time over which the alcohol was administered varied, depending on the complexity of the procedure. Schematic drawing about the procedure illustrated in Fig. 1.

The aim of treatment was symptom palliation and size reduction. Thus, sclerotherapy sessions were repeated until either the patient felt they could tolerate residual symptoms or the physician was unable to identify any further venous pouches to target with treatment. Patients were observed after the procedure for swelling, pain control, hematuria, and other complications.

\section{Evaluation of Clinical Data and Follow-up Results}

Patients were initially followed up at our department approximately $6-12$ weeks after the procedure. This time allowed resolution of swelling and changes directly related to sclerotherapy and proper assessment of the treatment results. Recommendations for repeat sclerosis or clinical follow-up were based on this initial assessment. Results of sclerotherapy were classified with subjective clinical assessment, including interview and examination. MR imaging follow-up was available for $4(18.2 \%)$ of 22 patients but was not included in the evaluation of clinical results. An interventional neuroradiologist (D.C.S.) performed the assessments. Both lesion size and patient symptoms were assessed including pain, degree of residual functional impairment, and cosmetic deformity. Clinical follow-up after the last treatment session was performed in all patients and ranged from 1 to 63 months (mean, 13 months). Clinical outcomes were classified as follows: (a) little or no change; (b) moderate decrease in size and symptoms; and (c) markedly decreased in size with clinical obliter- ation. Complications included death, permanent sequelae including skin necrosis, nerve palsy and infection, requirement of major therapy including infection, and prolonged hospitalization. Minor complications included any transient adverse effect.

\section{RESULTS}

During the 37 direct puncture alcohol sclerotherapy (range, 1-5; mean, 1.7) in 22 patients, the amount of alcohol ranged from 2 to $18 \mathrm{~mL}$ (mean, $8.5 \mathrm{~mL}$ ) in a single session. When maximum amounts of alcohol were used, the total dose did not exceed $1.0 \mathrm{~mL} / \mathrm{kg}$ of body weight. No patient experienced alcohol toxicity.

Percutaneous alcohol sclerotherapy resulted in improvement or resolution of symptoms in 21 (95.5\%) of 22 patients. The mean follow-up duration was 13 months (range, 1-63 months). With regard to the clinical outcome of alcohol sclerotherapy, 9 of 22 patients (41\%) were markedly decreased in size, 12 $(54.5 \%)$ were moderately decreased, and $1(4.5 \%)$ had little change. A single session was performed in 16 patients $(72.7 \%)$; multi-staged sclerotherapy was required in 6 patients $(27.3 \%)$.

Two patients $(9.1 \%)$ of 22 patients had minor complications including paraesthesia of the lesion, and reversible ophthalmic venous thrombosis. However, these complications recovered completely with time. And there was no procedure-related mortality. The patients' clinical data, technical details, and clinical outcomes are summarized in Table 1.

\section{DISCUSSION}

VMs are a congenital vascular malformation, although they are not always evident at birth, with $40 \%$ of cases found in the head and neck region. These

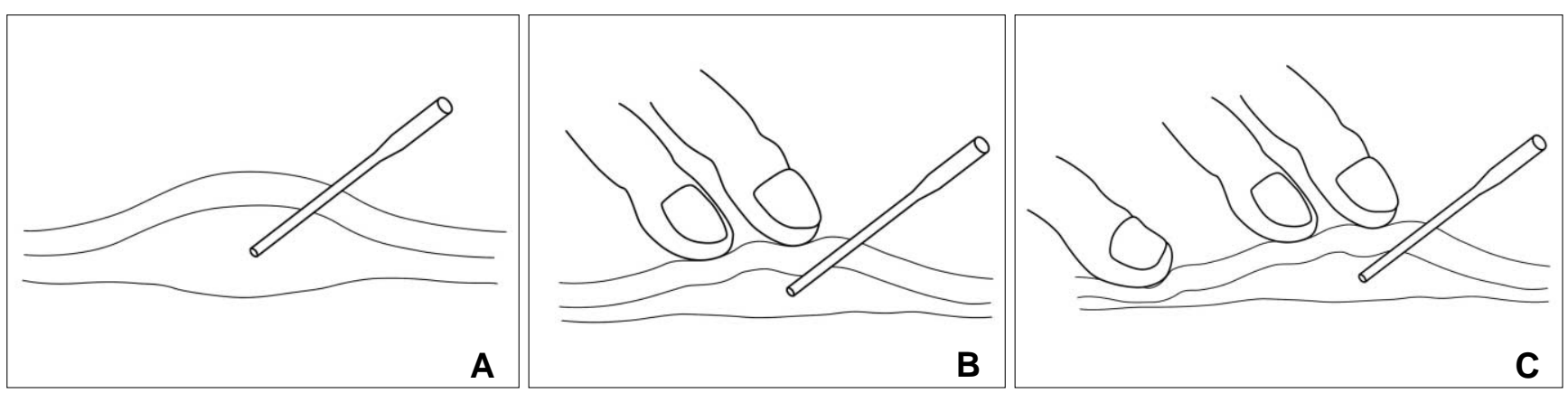

Fig. 1. Schematic procedural diagram of the compression technique during alcohol injection.

A. A needle tip is in the secured position in venous malformation of the subcutaneous area.

B. Venogram is obtained during compression and subsequent alcohol injection is done in the same position

C. Additional compression is required if there is any dangerous venous drainage. 
Percutaneous Alcohol Sclerotherapy for Venous Malformations

Table 1. Clinical Data, Technical Details, and Clinical Outcomes in 22 Patients with Venous Malformations

\begin{tabular}{|c|c|c|c|c|c|c|c|c|}
\hline \multirow{2}{*}{$\begin{array}{l}\text { Patient } \\
\text { No. }\end{array}$} & \multirow{2}{*}{$\begin{array}{l}\text { Sex/ } \\
\text { Age }\end{array}$} & \multirow{2}{*}{ Location } & \multirow{2}{*}{ Symptoms } & \multirow{2}{*}{$\begin{array}{c}\begin{array}{c}\text { No. } \\
\text { of } \\
\text { Procedures }\end{array}\end{array}$} & \multicolumn{2}{|c|}{ Complication } & \multirow{2}{*}{$\begin{array}{l}\text { Clinical } \\
\text { follow-up } \\
\text { (Months) }\end{array}$} & \multirow{2}{*}{$\begin{array}{l}\text { Clinical } \\
\text { Outcome }\end{array}$} \\
\hline & & & & & Major & Minor & & \\
\hline 1 & $M / 32$ & Submandibular & $S$ & 1 & No & No & 7 & E \\
\hline 2 & $M / 38$ & Neck & $S, P$ & 4 & No & No & 26 & E \\
\hline 3 & $\mathrm{M} / 17$ & Cheek, lip & $S$ & 4 & No & No & 63 & $G$ \\
\hline 4 & $M / 25$ & Tongue & S, D, I & 1 & No & No & 1 & $E$ \\
\hline 5 & $\mathrm{~F} / 54$ & Cheek & $\mathrm{S}, \mathrm{I}$ & 1 & No & No & 26 & E \\
\hline 6 & $F / 3$ & Neck & $S, P$ & 1 & No & No & 8 & $E$ \\
\hline 7 & $F / 39$ & Periorbital & $S, D$ & 1 & No & ROVT & 17 & $G$ \\
\hline 8 & $M / 8$ & Lip & S, D & 3 & No & No & 42 & $G$ \\
\hline 9 & $F / 17$ & Lip & S, I, D & 1 & No & No & 13 & $G$ \\
\hline 10 & $\mathrm{~F} / 30$ & Cheek & S, I, D & 5 & No & No & 13 & $G$ \\
\hline 11 & $M / 24$ & Lip & $S, D$ & 1 & No & No & 7 & $E$ \\
\hline 12 & $F / 41$ & Cheek & $S$ & 1 & No & No & 7 & $E$ \\
\hline 13 & $F / 43$ & Temporal & $S$ & 2 & No & No & 23 & NC \\
\hline 14 & $M / 23$ & Cheek & $S$ & 1 & No & No & 6 & $\mathrm{E}$ \\
\hline 15 & $F / 21$ & Cheek & $S$ & 1 & No & No & 6 & $E$ \\
\hline 16 & $F / 31$ & Lip & S, I, D & 1 & No & No & 1 & $G$ \\
\hline 17 & $\mathrm{M} / 45$ & Lip & S, B & 1 & No & No & 1 & $G$ \\
\hline 18 & $\mathrm{~F} / 14$ & Submandibular & S & 1 & No & No & 1 & $\mathrm{G}$ \\
\hline 19 & $F / 38$ & Lip & S, I, D & 1 & No & No & 6 & $\mathrm{G}$ \\
\hline 20 & $M / 29$ & Face & S, I & 3 & No & No & 9 & $\mathrm{G}$ \\
\hline 21 & $M / 28$ & Lip & $S, D$ & 1 & No & $\mathrm{Pa}$ & 1 & $\mathrm{G}$ \\
\hline 22 & $\mathrm{~F} / 67$ & Lip & D, B & 1 & No & No & 1 & $\mathrm{G}$ \\
\hline
\end{tabular}

Abbreviations: S, swelling; P, pain; D, discoloration; I, increase in size; B, bleeding; Pa, paresthesia; NC, no change; G, good: moderately decreased size; E, excellent: markedly decreased size; ROVT, reversible ophthalmic venous thrombosis.

lesions are "low-flow" vascular malformations that consist of dysmorphic channels lined by flattened endothelium and a thin basement membrane. They may become clinically apparent at any time throughout the lifespan, because they grow proportionately with age and do not regress [1-3].

Symptoms can have a wide range including pain, swelling, bluish-purple discoloration, infection, or bleeding. Functional impairment may be present, depending on the size, location, hemodynamic effects, and type of vessel involved. VM can be enlarged due to dilated venous channels during the Valsalva maneuver $[13,14]$.

Diagnosis is usually based on clinical presentation, with confirmation by MR imaging. VMs demonstrate septated soft tissue lesion with high signal intensity on T2-weighted images and phleboliths may be shown as multiple round signal intensity voids on MR images. Contrast administration results in a variable enhancement pattern, ranging from attenuated enhancement similar to that in adjacent veins to nonhomogeneous or delayed enhancement $[2,14]$.
Treatment of VMs is challenging but percutaneous alcohol sclerotherapy is the most common therapeutic option by means of direct lesional puncture. Among the sclerosing agents, alcohol is one of the most effective sclerosant which can induce denudation of endothelium, intense inflammatory reaction, and thrombosis of the VMs almost immediately $[1,5,7]$.

In this study, we administrated absolute alcohol under visual control, without using fluoroscopic guidance and without mixing contrast agent. And we also used manual compression of the VM itself for preventing adverse embolic effect of alcohol and evaluating the course of injected alcohol beyond the venous pouch during the procedure. We found that this method can be helpful to increase sclerotic effect and to reduce the total amount of alcohol and complications for treating VMs. Our results showed that moderate or markedly decreased size of the lesion resulted in $21(95.5 \%)$ of 22 patients overall; these findings appear to be similar or higher rate than those in the previous studies $[4,11$, 14]. Despite of only visual control during alcohol injection without using fluoroscopic guidance, there 
was no additional risk about an occurrence of complications. Our results showed that only two of 22 patients had minor complications, and there was no procedurerelated mortality. Compared to previous reports $[1,4$, $10,11]$, total amount of alcohol for sclerosis of the lesion could be markedly reduced by using this technique. A decreased amount of alcohol may also reduce local or systemic complications related to alcohol toxicity. In addition, position of the operator was comfortable because biplane fluoroscopy would not be necessary. However, in case of venous malformation with rapid outflow drainage, the intermittent fluoroscopic guidance should be performed to control rapid flow during the entire procedure.

Our study had several limitations. First, our study did not include large number of patients. Further risk that we could not detect can be identified. Therefore, careful application of this technique by experts is mandatory. Second, because there is no standard report form for VMs, we used our own criteria to assess the clinical outcome; this can make it difficult to compare our results with those from other studies. Furthermore, MR imaging follow-up was available for 4 patients, and we did not include follow-up MR images in the evaluation of clinical results. Follow-up MR imaging can be helpful to provide more objective information for evaluating clinical outcomes.

In conclusion, direct puncture alcohol sclerotherapy without fluoroscopy guidance can be a safe and effective technique for treating VMs of the head and neck areas.

\section{References}

1. Johnson PL, Eckard DA, Brecheisen MA, Girod DA, Tsue TT. Percutaneous ethanol sclerotherapy of venous malformations of the tongue. AJNR Am J Neuroradiol 2002;23:779-782

2. Spence J, Krings T, terBrugge K, da Costa L, Agid R. Percutaneous sclerotherapy for facial venous malformations: subjective clinical and objective MR imaging follow-up results. AJNR Am J Neuroradiol 2010;31:955-960

3. Legiehn GM, Heran MKS. Venous malformations: classification, development, diagnosis, and interventional radiologic management. Radiol Clin North Am 2008;46:545-597

4. Do YS, Yakes WF, Shin SW, Lee BB, Kim DI, Liu WC, et al. Ethanol embolization of arteriovenous malformations: interim results. Radiology 2005;235:674-682

5. Lee HY, Kim SM, Choi JW, In HS, Jang YJ, Cho KJ, et al. The Significance of immunohistochemical staining, including that for glucose transporter protein isoform 1, as related to the clinical and angiographic features of adult soft-tissue hemangioma and arteriovenous malformation in the head and neck. J Korean Soc Radiol 2009;60:83-91

6. Yakes W, Luethke J, Parker S, Stavros A, Rak K, Hopper K, et al. Ethanol embolization of vascular malformations. Radiographics 1990;10:787-796

7. Berenguer B, Burrows PE, Zurakowski D, Mulliken JB. Sclerotherapy of craniofacial venous malformations: complications and results. Plast Reconstr Surg 1999;104:1-11

8. Mason KP, Michna E, Zurakowski D, Koka BV, Burrows PE. Serum ethanol levels in children and adults after ethanol embolization or sclerotherapy for vascular anomalies. Radiology 2000;217:127-132

9. Yakes W, Haas D, Parker S, Gibson M, Hopper K, Mulligan J, et al. Symptomatic vascular malformations: ethanol embolotherapy. Radiology 1989;170:1059-1066

10. Hammer FD, Boon LM, Mathurin P, Vanwijck RR. Ethanol sclerotherapy of venous malformations: evaluation of systemic ethanol contamination. J Vasc Interv Radiol 2001;12:595-600

11. Fan X, Su L, Zheng J, Zheng L, Zhang Z. Ethanol embolization of arteriovenous malformations of the mandible. AJNR Am J Neuroradiol 2009;30:1178-1183

12. Goyal M, Causer PA, Armstrong D. Venous vascular malformations in pediatric patients: comparison of results of alcohol sclerotherapy with proposed MR imaging classification. Radiology 2002;223:639-644

13. Ryu C, Whang S, Suh D, Kim SM, Jang YJ, Kim HJ, et al. Percutaneous direct puncture glue embolization of high-flow craniofacial arteriovenous lesions: a new circular ring compression device with a beveled edge. AJNR Am J Neuroradiol 2007; 28:528-530

14. Mulliken JB, Glowacki J. Hemangiomas and vascular malformations in infants and children: a classification based on endothelial characteristics. Plast Reconstr Surg 1982;69:412-422

15. Gandhi D, Gemmete J, Ansari S, Gujar S, Mukherji S. Interventional neuroradiology of the head and neck. AJNR Am J Neuroradiol 2008;29:1806-1815 